\title{
CLASSIFICATION DES SCIENCES ET CLASSIFICATION DES PLANTES CHEZ AUGUSTIN-PYRAMUS DE CANDOLLE
}

\author{
Jean-Marc DrouIN
}

Resume : Dans la Théorie élémentaire de la botanique, dont la première édition date de 1813, Augustin-Pyramus de Candolle se propose d'expliquer et de fonder rationnellement la méthode naturelle de classification des plantes. Pour cela, il est amené à comparer les différentes méthodes existantes. Par ailleurs, dans l'introduction, il propose une classification générale des sciences. Dans les deux cas, on retrouve un même souci de justifier le statut scientifique de la botanique.

L'exemple de l'histoire naturelle est souvent évoqué dans les débats sur les classifications ${ }^{1}$. À cet égard la Théorie élémentaire de la botanique d'Augustin-Pyramus de Candolle, parue en 1813, mérite doublement de retenir l'attention: non seulement elle constitue une des tentatives les plus significatives pour fonder rationnellement la taxonomie végétale, mais de plus elle s'ouvre par une classification des sciences ${ }^{2}$. Suffit-il cependant qu'elles soient ainsi réunies sous la même plume, dans le même volume, pour que les deux classifications révèlent quelque parenté profonde qui rapprocherait leur démarche par-delà l'hétérogénéité manifeste de leurs objets? Ou bien faut-il chercher ailleurs, dans une communauté d'inspiration, le lien qui les unit ? Ou encore renoncer à toute idée de filiation et ne voir là qu'une simple coexistence de deux exercices intellectuels?

Augustin-Pyramus de Candolle naît en 1778 dans une famille protestante d'origine française établie à Genève dès le $x^{e}{ }^{e}$ siècle $^{3}$. Encore ado-

1. Auguste Comm, Cours de philosophie positive, $2^{c}$ leçon, 1830, Paris, Hermann, 1975, p. 44 : « La théorie générale des classifications, établie dans ces derniers temps par les travaux philosophiques des botanistes et des zoologistes permet d'espérer un succès réel dans un semblable travail, en nous offrant un guide certain par le véritable principe fondamental de l'art de classer, qui n'avait jamais été conçu distinctement jusqu'alors. "

2. Augustin-Pyramus de CANDOLLE, Théorie élémentaire de la botanique ou exposition des principes de la classification naturelle et de l'art de décrire et d'étudier les végétaux, $1^{\text {to }}$ éd., Paris, Déterville, $1813 ; 2^{c}$ éd., Paris, Déterville, $1819 ; 3^{c}$ éd., Paris, Roret, 1844.

3. Sur A.-P. de Candolle, on trouvera, in Franz A. Stafleu, Richard S. Cowan, Taxonomic Literature, vol. 1, Utrecht, Scheltema et Holkema, 1976, p. 438-452, une liste d'articles antérieurs à 1972. Parmi les publications plus récentes à caractère biographique, on peut noter Revue de synthèse : IV S. N ${ }^{\text {os }} 1-2$, janv.-juin 1994. 
lescent, il herborise dans le Jura suisse et se prend de passion pour la botanique. Durant l'hiver 1797-1798, ses parents l'envoient à Paris compléter son éducation et c'est dans cette ville qu'il s'installe lorsqu'au printemps 1798, Genève est officiellement "réunie " à la France. Il publie ses premiers travaux et fréquente divers cercles scientifiques, dont la Société philomathique et la Société d'Arcueil. Après plusieurs tentatives infructueuses pour entrer à l'Institut, il accepte, en 1808, un poste à l'université de Montpellier. C'est là, loin de la capitale mais cependant dans une terre d'élection de la médecine et de la botanique, qu'il rédige la Théorie élémentaire.

En le chassant de Montpellier et en le ramenant à Genève, les événements des années 1814-1815 ouvrent une nouvelle période dans sa vie. Alors qu'il est en butte à l'hostilité des ultras qui lui reprochent d'avoir été recteur durant les Cent-Jours, Genève, redevenue indépendante grâce à la chute de l'Empire et à son rattachement à la Confédération helvétique, lui offre une chaire d'histoire naturelle. Il accepte et prend une part active à la vie politique, sociale et mondaine de sa ville natale. En même temps, il s'attelle à une tâche qu'il projette depuis longtemps : décrire et classer toutes les espèces végétales connues. Cette entreprise évoque celle de Linné au siècle précédent, mais la découverte en grand nombre de nouvelles espèces lui donne un caractère démesuré. Avec une modestie résignée, de Candolle intitule "ébauche", Prodromus, ce tableau du règne végétal dont le premier volume paraît en $1824^{4}$. Malgré de nombreuses collaborations, huit volumes seulement, soit environ la moitié du total, seront parus à sa mort en 1841 et c'est son fils, Alphonse de Candolle, lui-même botaniste, qui prendra le relais ${ }^{5}$.

Outre son ceuvre scientifique, Augustin-Pyramus de Candolle est l'auteur de Mémoires et souvenirs publiés en 1862 avec quelques coupures et quelques documents annexes ${ }^{6}$. Cette autobiographie a retenu l'attention non seulement des botanistes mais aussi de tous ceux qui se sont intéressés au milieu scientifique genevois et à sa spécificité ${ }^{7}$. Les

les études suivantes : Roger de CANDolle, "Augustin-Pyramus de Candolle et Genève", Musée de Genève, vol. 19, 182, 1978, p. 9-12 ; Pierre Chevrot, "Augustin-Pyramus de Candolle et la pédagogie ", ibid., p. 13-16; Jacques MIEGE, « Augustin-Pyramus de Candolle : sa vie, son cuuvre, son action... ", Mémoires de la Société de physique et d'histoire naturelle de Genève, vol. 43, 1, 1979, p. 1-45.

4. A.-P. de CANDOlle et al., Prodromus systematis regni vegetabilis, Paris, Treuttel et Wurtz, 1824.

5. Cf. Jean-Marc DroulN, « Botanique et sciences sociales chez Alphonse de Candolle ", Corpus, revue de philosophie, 7, 1988, p. 155-163.

6. A.-P. de CANDOLle, Mémoires et souvenirs de Augustin-Pyramus de Candolle écrits par lui-même et publiés par son fils, Genève, Jö̉l Cherbuliez, 1862.

7. Sur ce thème, voir : Cléopâtre Montandon, Le Développement de la science à Genève aux xvII' et XIX' siècles, Vevey, Delta, 1975; Les Savants genevois dans l'Europe intellectuelle 
anecdotes, les scènes de la vie quotidienne y foisonnent. L'une d'elles illustre aux yeux du narrateur, son intérêt précoce pour les problèmes de classification :

« Je me rappelle assez distinctement qu'il y avait dans la propriété de mon père une grande variété de fruits ; ma mère m'avait chargé de ranger son fruitier; je le faisais avec plaisir et avec soin, et je donnais une attention singulière à ne pas confondre les espèces et à les combiner de manière à mettre à côté des autres les espèces qui se ressemblaient. On se moquait de mes minuties, c'était cependant déjà un indice de mes goûts futurs, on me disait qu'en plaçant les unes à côté des autres les espèces qui se ressemblaient le plus, elles devenaient plus difficiles à distinguer; je répondais que je devais les ranger dans le fruitier d'après leurs ressemblances naturelles; je préférais par instinct la méthode naturelle à la méthode artificielle ${ }^{8}$.

Ce souvenir d'enfance a valeur de fable didactique. Le rangement commode suggéré par les parents représente les méthodes artificielles préconisées par la plupart des botanistes de la vieille génération; la minutie, avec laquelle le jeune de Candolle dispose les fruits en respectant leurs véritables affinités, préfigure à ses yeux son œuvre de systématicien. Celle-ci est en effet tout entière animée du souci de montrer la valeur scientifique d'une classification fondée sur la "méthode naturelle " et sa supériorité sur les classifications artificielles, quels que puissent être les avantages pratiques de ces dernières.

De ce point de vue, la réédition de la Flore française de Lamarck par de Candolle revêt une importance singulière. Celle-ci, parue en 1778 avait été réimprimée en $1795^{\circ}$. Une troisième édition s'imposait, elle impliquait un travail considérable de révision tenant compte à la fois des progrès de la botanique et de l'annexion à l'entité française de nouveaux territoires. C'est précisément ce travail qu'a réalisé de Candolle à la demande de Lamarck.

du xVIf au milieu du XIx siècle, dir. J. Trembley, Genève, Éditions du Journal de Genève, 1987. Pour une bibliographie et une mise au point récente : René SigrisT, Les Origines de la Société de physique et d'histoire naturelle (1790-1822). La science genevoise face au modèle français, Genève, S.P.H.N./Muséum de Genève, 1990.

8. A.-P. de CaNDOlle, op. cit. supra n. 6, p. 12. Cette anecdote n'est pas datée précisément. Dans la narration, elle se place entre une maladie qui met en danger les jours d'Augustin-Pyramus, alors âgé de sept ans, et son entrée au collège à onze ans. Quant à sa date de rédaction, on peut supposer que l'auteur a écrit en premier ces premières pages de ces mémoires, donc en 1821, ou peu après puisque c'est la date à laquelle il dit avoir commencé à rédiger.

9. Jean-Baptiste de Monet, chevalier de LAMARCK, Flore françoise..., 3 vol., Paris, Imprimerie royale, 1778; ID., ibid., 3 vol., $2^{c}$ éd., Paris, Agasse, an III, 1795. 
L'ouvrage paraît en 1805 , il comprend cinq volumes au lieu de trois pour l'édition de $1778^{10}$. Pour le présenter au public, de Candolle rédige une lettre-préface adressée à Lamarck qui lui permet de marquer sa fidélité au projet initial tout en signalant ses innovations ${ }^{11}$.

La Flore de Lamarck offre au lecteur, d'une part, une initiation aux principes généraux de la botanique et, d'autre part, une description des espèces végétales qu'on peut trouver en France avec une méthode pour les reconnaître facilement. De Candolle s'en réjouit puisqu'il considère comme complémentaires et d'une égale importance "la connaissance générale des organes et des fonctions des végétaux » et "l'art de distinguer les végétaux les uns des autres " ${ }^{12}$.

Or, pour distinguer les plantes les unes des autres, explique de Candolle, deux méthodes, deux routes dit le texte, se présentent : l'une, naturelle, cherche une classification qui rende compte des ressemblances les plus importantes entre les espèces, l'autre artificielle, cherche un moyen d'identifier chaque plante et de trouver son nom. La première est une "véritable science », la seconde un « art empirique », autrement dit une technique. Pour atteindre à la vérité, une classification naturelle se base « sur les organes les plus importants à la vie des végétaux, sans considérer si ces organes sont faciles ou difficiles à observer " ${ }^{13}$. En revanche, pour remplir son but qui est d'être commode, une méthode de détermination ne doit prendre en compte que les caractéristiques les plus faciles à observer. Lamarck est le premier, dit de Candolle, à avoir distingué ces deux objectifs et proposé une méthode de détermination qui annonce clairement son caractère artificiel ${ }^{14}$. Cette méthode, aujourd'hui familière aux amateurs de botanique, Lamarck l'appelle « analyse », de Candolle "méthode analytique». Elle permet de trouver le nom de n'importe quelle plante décrite dans la Flore par une série de questions à deux réponses contradictoires.

10. Un sixième volume est paru en 1815 . Cette date de 1815 se retrouve sur certains exemplaires des premiers volumes; en effet, d'après une note d'Alphonse de Candolle dans les Mémoires et souvenirs de son père, op. cit. supra n. 6, p. 497, « le libraire, sans consulter l'auteur, a vendu des exemplaires avec un titre portant pour tous les volumes la date du der. nier $"$.

11. A.-P. de Candolle, "A Mr de Lamarck... ", in J.-B. de Lamarck et A.-P. de CanDOLLE, Flore françoise..., Paris, Deray, 1805, t. 1, p. vi-x.

12. Ibid., p. vii.

13. Ibid., p. vii.

14. Ibid., p. viii. Michel Foucaut, in Les Mots et les choses, Paris, Gallimard, 1966, p. 243, a soutenu que Lamarck, par cette distinction, aurait " entrouvert l'âge de la biologie " "d'une façon bien plus certaine et radicale " que par ses idées sur la transformation des espèces. Le paradoxe repose sur une appréciation discutable du transformisme lamarckien mais il a le mérite de souligner la portée théorique du problème soulevé par Lamarck en 1778 et développé ici par de Candolle. 
Jusque-là de Candolle se fait assez fidèlement l'écho du « Discours préliminaire " dans lequel Lamarck, peut-être avec l'aide d'autres naturalistes, exposait les principes de son livre et en soulignait la nouveaute ${ }^{15}$. Toutefois, il n'y a pas identité complète de vue entre les deux auteurs, et ceci apparait quand est annoncé le plan de l'ouvrage. Dans l'édition originale en 1778 , l'analyse occupe la plus grande part de l'ouvrage et la disposition des espèces dans un tableau qui montre leurs rapports et leurs affinités n'est qu'ébauchée en quelques pages. Dans la réédition de 1805 , la méthode analytique - autrement dit l'ensemble des clés de détermination - tient en un seul volume et le reste de l'ouvrage contient les descriptions groupées en familles naturelles selon la méthode de Jussieu. De Candolle combine ainsi, à l'aide d'un système de renvoi et de codage, la méthode artificielle "destinée à faire connaitre le nom des plantes » et la méthode naturelle qui rend compte de « la structure ", de «l'histoire " et des « rapports de ces mêmes plantes " ${ }^{16}$.

\section{CLASSER LES CLASSIFICATIONS ELLES-MÊMES}

Les idées, esquissées en quelques pages en 1805 , se retrouvent en 1813 développées dans les cinq premiers chapitres de la Théorie élémentaire.

De Candolle rappelle tout d'abord le nombre immense des espèces végétales connues, trente mille à l'époque, et la nécessité d'un guide pour s'orienter dans ce "dédale effrayant " ${ }^{17}$. Sans prétendre tout connaître d'un végétal, nous pouvons espérer « trouver ce que les autres hommes en ont su " et être «à même de savoir si ce que nous observons l'a déjà été par quelqu'un d'autre " ${ }^{18}$. Pour cela nous devons disposer d'une méthode qui, après avoir divisé en plusieurs groupes le règne végétal, nous permette de trouver la plante qui nous intéresse. Pour désigner cette "partie de l'étude des végétaux ", de Candolle propose le terme de " taxonomie", qu'il semble être le premier à avoir utilisé, et souligne la complémentarité de celle-ci avec la " physiologie ${ }^{19}$. Il insiste ensuite sur

15. D’après Daudin, le "Discours préliminaire " aurait été composé par Lamarck certes mais sous la direction de Daubenton et avec l'aide d'Haüy, cf. Henri DAudin, De Linné à Lamarck. Méthodes de la classification, Paris, Alcan, 1926-1927, réimpr. Paris, Éditions des Archives contemporaines, 1983, p. 191.

16. Voir op. cit. supra n. 11, p. viii.

17. Op. cit. supra n. 2,1813 , p. 23.

18. Ibid., p. 24.

19. Ibid. Sur «taxonomie », voir Jean-Louis FISCHER, Roselyne REY, « De l'origine et de l'usage des termes taxinomie-taxonomie », Documents pour l'étude du vocabulaire scientifique (Paris, CNRS), 5, 1983, p. 97-113. 
le grand nombre de classifications proposées par les botanistes depuis la Renaissance, au point dit-il, que même simplement pour les indiquer « il est nécessaire de classer les classifications " ${ }^{20}$.

Une première distinction oppose les classifications empiriques et les classifications rationnelles. Les premières sont "indépendantes de la nature de l'objet "; elles se réduisent, par exemple, à une disposition par ordre alphabétique comme celle de certains livres de plantes au $x^{e}{ }^{e}$ siècle. Les secondes sont rationnelles dans le sens où elles « ont un rapport réel avec les objets auxquels on les applique ». Ces classifications rationnelles se divisent elles-mêmes en trois groupes selon le but qu'elles se proposent. Le premier groupe contient les classifications " usuelles " (ou "pratiques ") fondées sur les usages des végétaux, par exemple sur leurs propriétés thérapeutiques. Les deuxième et troisième groupes correspondent l'un aux méthodes artificielles, destinées à faire connaître le nom des plantes, l'autre aux méthodes naturelles, visant à faire connaitre les rapports réels entre les espèces. En fait, malgré la mention préalable des classifications « empiriques " et " usuelles ", l'attention se centre sur les catégories distinguées en 1805, classifications artificielles et classifications naturelles, et sur la nécessité de ne pas les confondre ${ }^{21}$.

Parmi les classifications artificielles, de Candolle place celle de Tournefort. Celui-ci conservait la vieille distinction entre les herbes et les arbres, puis divisait chacun de ses groupes d'après la forme de la corolle. Plusieurs pages sont également consacrées au système de Linné fondé sur le nombre des étamines et des styles. Il reproduit même le tableau complet des classes linnéennes. Tout ceci n'a plus pour lui qu'un intérêt historique car la classification proposée par Linné et que défendent encore certains botanistes à son époque, lui paraît devoir être abandonnée ${ }^{22}$. Ceci n'entame d'ailleurs pas l'admiration qu'il professe pour Linné, dont, comme l'ensemble des naturalistes, il utilise évidemment la nomenclature $^{23}$. En définitive, et malgré un ton plus réservé que dans sa lettrepréface de 1805 , il donne la préférence parmi les méthodes artificielles à celle de Lamarck, parce que, précisément, elle est ouvertement artificielle. En même temps, de Candolle engage les débutants à ne pas s'en contenter, s'ils veulent vraiment pratiquer la botanique comme une activité scientifique et non comme une simple énumération de noms.

20. Op. cit. supra n. 2, p. 25-26.

21. Ibid., p. 26-27.

22. Sur les linnéens tardifs, voir Pascal DuRIs, Linné et la France. 1780-1850, Genève, Droz, 1993.

23. La nomenclature linnéenne d'un usage universel encore aujourd'hui désigne, en latin, chaque espèce par un nom de genre, qu'elle partage avec les espèces voisines, auquel s'ajoute l'épithète propre à l'espèce. 
En définitive, c'est aux méthodes naturelles qu'est lié le progrès de la discipline et c'est à elle surtout que de Candolle s'intéresse ici. Sans prétendre les énumérer toutes, il entend faire sentir leur diversité et, pour cela, il en distingue trois sortes, " celles de tâtonnement, de comparaison générale et de subordination des caractères ${ }^{24}$.

1) Dans le premier cas, celui du tâtonnement, c'est par ajustement successifs, sans règle a priori, qu'on cherche à disposer les plantes en se rapprochant de l'ordre naturel. Parmi les auteurs qui ont procédé ainsi, on trouve Pierre Magnol, directeur du jardin botanique de Montpellier au $\mathrm{xvII}^{\mathrm{e}}$ siècle. Son ouvrage témoigne que les anciens botanistes avaient senti l'existence de rapports naturels entre les espèces mais qu'en l'absence d'une "règle pour rechercher ces rapports", les uns pouvaient regarder deux plantes comme analogues tandis que d'autres pouvaient les séparer comme dissemblables, "sans qu'aucune des opinions pût être prouvée vraie ou fausse $"{ }^{25}$. Est ensuite évoquée la tentative de Linné pour disposer les plantes dans un ordre naturel. Pour de Candolle, c'est parce que Linné en est resté dans ce domaine à un tâtonnement infructueux, qu'il a renoncé à la méthode naturelle et a préféré proposer le système artificiel évoqué précédemment.

2) Le deuxième principe sur lequel peut se fonder une méthode naturelle est celui de comparaison générale. L'exemple le plus illustre en est fourni par l'œuvre de Michel Adanson. De Candolle résume ainsi la démarche de l'auteur des Familles des plantes (1763):

« ayant pensé avec raison que toutes les parties des plantes devaient être prises en considération dans la méthode naturelle, il établit, sur chaque organe des plantes pris séparément, un ou plusieurs systèmes déduits de leur situation, de leur figure, de leur nombre, de leur proportion, de leur durée et de leur substance : il résulta de cette entreprise la formation de 65 systèmes artificiels; après cet immense travail, Adanson pensa que les plantes qui se trouvaient les unes à côté des autres dans le plus grand nombre de ces systèmes, devaient être celles qui avaient entr'elles le plus de rapports, et qu'on devait le plus rapprocher dans l'ordre naturel $"{ }^{26}$.

À cette idée «séduisante au premier coup d'œil par son exactitude apparente ", de Candolle oppose une double objection : d'une part, nous ne connaissons pas tous les organes des plantes et tous les points de vue

24. A.-P. de Candolle, op. cit. supra n. 2, p. 67.

25. Ibid., p. 68.

26. Ibid., p. 70. Pour la présentation des 65 systèmes, voir Michel Adanson, Familles des plantes, 2 vol., Paris, 1763 , p. ccii-cciii. En réalité, ce n'est là pour Adanson qu'une reconstruction a posteriori. La démarche par laquelle il a élaboré sa méthode est un peu différente, mais elle repose aussi sur la prise en compte de tous les caractères de la plante. 
sous lesquels il faut les considérer, d'autre part, tous ces organes n'ont pas la même importance, certains ont une plus grande influence que d'autres sur l'organisation et la conservation de la plante.

3) Le troisième principe possible pour une méthode naturelle, celui de la subordination des caractères, répond à cette constatation que certains organes ont plus d'importance que d'autres et qu'il importe donc d'établir « la classification d'abord sur ces organes prédominants, puis les

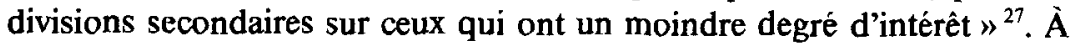
cette méthode de classification, qui revient à pondérer les caractères afin d'imiter "l'ordre de la nature », est attaché le nom des Jussieu. De Candolle évoque Bernard de Jussieu, qui avait disposé le jardin du Trianon suivant cette méthode, puis son neveu, Antoine-Laurent de Jussieu, qui, en publiant en 1789 le Genera plantarum, a réalisé, dans « les sciences d'observation " une révolution comparable à celle que Lavoisier opère à la même époque "dans les sciences d'expérience " ${ }^{28}$.

Dans ces « Considérations préliminaires sur les classifications en général ", de Candolle ne développe pas plus avant les principes de la méthode naturelle mais il consacre tout le reste de la première des trois parties que comporte l'ouvrage à les exposer, à les expliquer, à les défendre et à les développer ${ }^{29}$. Démarche classique au demeurant que celle qui consiste à présenter sa méthode en la comparant aux autres et à préparer ainsi le lecteur à la considérer comme la meilleure.

De manière analogue, mais plus inattendue peut-être, cette préparation est elle-même précédée d'une introduction dans laquelle de Candolle compare la botanique aux autres sciences et, pour ce faire, propose une classification complète des savoirs.

\section{LA DIVISION LA PLUS NATURELLE DES CONNAISSANCES HUMAINES}

Cette classification des sciences commence par une partition qui est opérée dès le premier paragraphe de l'introduction :

27. A.-P. de Candolle, op. cit. supra n. 2, p. 73.

28. Georges Cuvier, Rapport à l'Empereur sur les progrès des sciences..., citè par A.-P. de Candolle, in op. cit. supra n. 2, p. 74.

29. Sur ce point, cf. François DaGoGner, « Valentin Haüy, Étienne Geoffroy Saint-Hilaire, Augustin-P. de Candolle : une conception d'ensemble mais aussi un ensemble de conceptions ", Revue d'histoire des sciences, vol. 25, 4. 1972, p. 327-336; Peter F. Stevens, « Haüy and A.P. de Candolle, Cristallography, Botanical Systematics and Comparative Morphology, 1780-1840 ", Journal of the History of Biology, vol. 17, 1, 1984, p. 49-82; Delfo TeSI, « Augustin-Pyramus de Candolle : essai d'élaboration d'une taxonomie théorique au xix siècle », Gesnerus, vol. 39, 2, 1982, p. 295-303. 
«Quelque nombreuses que soient les branches des connaissances humaines, quelque variés que paraissent être les moyens que nous avons pour parvenir à la vérité, on doit les réduire à trois grandes classes : le raisonnement, le témoignage des autres hommes et l'expérience acquise par nos propres sensations ; d'où résulte la division la plus naturelle des connaissances humaines en sciences rationnelles, testimoniales et expérimentales $"{ }^{30}$.

De Candolle précise ensuite que la «manière d'opérer et de raisonner " d'une discipline ainsi que le " genre de certitude dont elle est susceptible" seront différents selon qu'elle appartiendra aux "sciences rationnelles ", aux "sciences testimoniales ou historiques" ou aux "sciences expérimentales»:

\begin{abstract}
«Les sciences rationnelles, telles que la logique, les mathématiques, sont tout entières produites par notre entendement, et peuvent exister indépendamment de l'existence ou de la connaissance de tous les êtres. Les sciences testimoniales ou historiques reposent essentiellement sur le témoignage des hommes dont le raisonnement pèse et discute la valeur. Les sciences expérimentales s'aident beaucoup du raisonnement et du témoignage des hommes ; mais elles ont ceci de particulier, que dans chaque cas, tout individu qui en a la volonté, peut à la rigueur s'en passer, ou du moins s'assurer, par le témoignage de ses propres sens, de la vérité des faits que le raisonnement ou le témoignage d'autrui lui ont fait connaitre ${ }^{31}$.
\end{abstract}

On le voit, le rapport entre les trois " moyens de parvenir à la vérité " et les trois classes de "connaissances humaines " est loin d'être univoque. Les sciences rationnelles n'ont besoin que du raisonnement. En revanche, les sciences testimoniales ou historiques, qui reposent sur le témoignage des hommes, ne peuvent se passer du raisonnement pour tirer parti de ce témoignage. Enfin, dans les sciences expérimentales, le raisonnement et le témoignage des hommes peuvent toujours être corroborés par l'expérience sensible, mais cette possibilité même prouve qu'ils ne sont pas exclus. La double conclusion que de Candolle ne tire pas luimême mais qui se déduit de cette conception est que le raisonnement peut se passer des autres modes de connaissance tandis que ceux-ci ne peuvent se passer de lui - ce qui explique le privilège des mathématiques et de la logique - mais qu'inversement la logique et les mathématiques font appel à une seule source de connaissance, alors que l'histoire en fait intervenir deux et les sciences expérimentales trois.

30. A.-P. de CAndolle, op. cit. supra n. 2, p. 1.

31. Ibid., p. 1-2. 
De Candolle ne donne d'ailleurs aucune précision sur la logique et les mathématiques, pas plus que sur l'histoire; en revanche, il s'attache, on ne s'en étonnera pas, aux sciences expérimentales. Il en distingue trois principales - l'histoire naturelle, la physique et la chimie - et souligne leur complémentarité.

À cette deuxième division s'en ajoute une troisième, puisqu'à l'intérieur de l'histoire naturelle il importe d'opérer une distinction " fondée sur les différences générales que les corps de la nature ont entre eux » :

" Ainsi, dès le premier coup d'œeil, on a vu que les uns présentaient une certaine disposition particulière, telle que chacune de leurs parties remplit un emploi spécial, et que de leur ensemble, résulte l'existence du tout: on a nommé ces parties agissantes, des organes; les corps dans lesquels on les aperçoit des corps organisés ou des êtres; et la science qui s'occupe de leur étude, l'histoire naturelle organique. On a vu, qu'outre ces êtres, il existait d'autres corps dans lesquels les parties ne sont ni distinctes entr'elles, ni chargées d'emplois particuliers : on les a nommés corps inorganisés, corps bruts ou simplement corps; et la science qui les étudie, est l'histoire naturelle inorganique $"{ }^{32}$.

Cette troisième division en entraîne à son tour deux autres, puisque l'histoire naturelle inorganique se scinde elle-même en deux :

" La science qui s'occupe des corps inorganiques, considérés comme partie du monde, est l'Astronomie. Celle qui les étudie, en tant que constituant le globe terrestre, peut recevoir le nom de Géonomie, étude immense dont la Géographie physique, la Météorologie, la Minéralogie, l'Oryctologie [étude des fossiles], la Géologie, sont les branches principales " ${ }^{33}$.

L'histoire naturelle organique, de son côté, est divisée en Zoologie et Botanique selon qu'elle s'occupe des animaux définis comme des êtres « doués de sentiment, c'est-à-dire de la conscience de leur existence » ou des végétaux, "dépourvus de toute sensibilité ". La botanique elle-même comprend deux parties : " la botanique proprement dite » d'une part, la " physique végétale " d'autre part, qui comprennent à leur tour chacune trois ou quatre disciplines.

Au milieu de cette classification et pour justifier la distinction des êtres organisés et des corps bruts, de Candolle s'efforce de caractériser, par contraste, la constitution des uns et des autres :

« Les corps bruts ne devant leur existence qu’à la réunion des molécules intégrantes, la forme, la masse et surtout la nature de celles-ci, seront donc

32. Ibid., p. 2-3.

33. Ibid., p. 3-9. 
véritablement les caractères essentiels de chacun de ces corps. Au contraire dans les corps organisés, les molécules intégrantes ne détermineront point la nature d'un être, mais ce sera la disposition générale de ces molécules ou la forme de ses parties, qui sera l'objet essentiel à étudier ${ }^{34}$.

S'ils s'opposent par leurs structures, les deux types de corps, organiques et inorganiques, sont, en ce qui concerne les lois auxquelles ils sont soumis, dans une relation inclusive :

« Les corps bruts sont entièrement soumis aux lois de l'attraction, et par conséquent aux principes simples de la physique et de la chimie ; les corps organisés sont, comme les autres, soumis à toutes ces lois et en outre à celles de la force vitale, qui suspend ou modifie, selon les cas, les lois ordinaires de la matière $"{ }^{35}$.

Ainsi, la classification des sciences s'accompagne, très explicitement, d'une réflexion philosophique sur la connaissance et le mode d'être de ses objets. Nous ne connaissons pas toutes les sources de cette réflexion mais l'une d'entre elles est suggéree par de Candolle dans ce passage des Mémoires et souvenirs :

« J'entrais en philosophie [...]. La logique et la métaphysique étaient enseignées par M. Prévost, homme exact, profond et spirituel, qui au moyen des connaissances très variées qu'il possédait, savait donner à ces études, ordinairement arides, un grand intérêt. [...] Je ne sais si ces études se trouvaient en harmonie avec ma tournure d'esprit ou si elles ont développé en moi des habitudes logiques, mais il est certain qu'elles ont souvent été depuis lors le sujet de réflexions utiles, et je n'ai point douté qu'une partie du succès de ma Théorie élémentaire ne soit due à l'influence des cours de M. Prévost ${ }^{36}$.

Or ce «M. Prévost» qui enseignait la philosophie à l'Académie de Genève n'est autre que Pierre Prévost, une des grandes figures de la vie intellectuelle genevoise de l'époque ${ }^{37}$. Juriste et helléniste, il est connu des historiens de la physique pour ses travaux sur la chaleur. En philosophie, outre ses traductions de l'Essai sur le principe de population de Malthus, des Essais philosophiques d'Adam Smith et des Éléments de la philosophie de l'esprit humain de Dugald Stewart, il est l'auteur d'Essais

34. Ibid., p. 7.

35. Ibid.

36. A.-P. de CANDOlle, op. cit. supra n. 6, p. 31-32. Si on en croit de Candolle tous les étudiants genevois ne partageaient pas son enthousiasme puisqu'il devait régulièrement prêter à ses condisciples ses résumés de cours.

37. Sur Pierre Prévost (1751-1839), cf. Les Savants genevois, op. cit. supra n. 7, p. 140-142 et 427 ; R. SigRIST, op. cit. supra n. 7, p. 41-42. 
de philosophie publié en 1804-1805 ${ }^{38}$. La lecture de cet ouvrage, qu'il présente lui-même dans la préface comme le cours qu'il professe depuis dix ans à Genève permet de se faire quelque idée de l'enseignement philosophique reçu par de Candolle.

En ce qui concerne la classification des sciences, le tableau de Bacon est présenté élogieusement par Prévost mais non sans quelques critiques. L'une d'elles porte sur la place des "arts mécaniques », rangés par Bacon sous le chef de la mémoire, alors que leur théorie « dépend de la raison, et leur pratique de l'habitude ". Par ailleurs, Prévost regrette qu'on inclue la poésie dans « le cercle des objets scientifiques " et souhaite qu'on lui donne un rang à part "convenable à sa dignité, et conforme au but qu'elle se propose ». Enfin, et c'est en réalité «le premier et le plus important » changement qu'il souhaite apporter à ce tableau, il veut distinguer les sciences selon qu'elles visent simplement à une vérité conditionnelle ou à une vérité absolue ${ }^{39}$. La «vérité conditionnelle », définie par l'identité entre le principe et la conséquence, ne relève que du raisonnement pur, et concerne les mathématiques. La " vérité absolue " est celle qu'on cherche à atteindre par les témoignages et l'expérience, c'est sur elles que se fondent les sciences historiques et les sciences expérimentales. Pour traduire la pensée de Prévost en d'autres termes, on pourrait dire que, dans les mathématiques, la pensée est en accord avec elle-même, tandis que, dans les sciences historiques ou expérimentales, elle est en quête d'une adéquation au réel. La classification proposée ensuite semble en retrait sur cette analyse, animée qu'elle est du souci de ne pas trop s'éloigner de celle de Bacon. Prévost distingue le " génie des arts», fondé sur l'imagination, et le « génie des sciences ", fondé sur l'entendement; à l'intérieur de ce dernier, on retrouve l'histoire qui correspond à la mémoire et la philosophie liée au raisonnement. L'histoire comprend l'histoire naturelle aussi bien que l'histoire des hommes ou la grammaire. La philosophie inclut les mathématiques et la physique raisonnée...

Cette classification ne pouvait pas être d'un grand secours à de Candolle, en revanche il est certain que la première division qu'il propose, entre sciences rationnelles, testimoniales et historiques, s'inscrit dans le droit fil des réflexions de son ancien maître, puisque cette tripartition est en fait une double dichotomie avec le raisonnement pur d'un côté et le témoignage et l'expérience de l'autre. Pour les divisions suivantes - à

38. Pierre Prevost, Essais de philosophie ou Étude de l'esprit humain, 2 vol., Genève, Paschoud, an XIII [1804-1805]. Le premier volume s'intitule Analyse des facultés de l'esprit humain et le second Logique.

39. Ibid., vol. 2, Partie II, Section I, chap. 1, p. 19 (la pagination n'est pas uniforme). 
l'intérieur des disciplines expérimentales - de Candolle s'est écarté de l'enseignement de Prévost, du moins tel qu'il apparaît dans les Essais, pour fonder la distinction de la physique, de la chimie et de l'histoire naturelle sur la distinction des corps inorganiques et des êtres organisés. Toujours est-il qu'il devait attacher une grande importance à cette question de la classification des sciences et ne pas être totalement satisfait de celle qu'il avait proposée, puisqu'en rééditant la Théorie élémentaire en 1819 , il éprouva le besoin de remanier l'introduction et d'aborder de manière sensiblement différente la classification des sciences.

\section{SCIENCES MATERIELLES ET SCIENCES DYNAMIQUES}

La première division est la même que dans l'édition de 1813 - sciences rationnelles, testimoniales, expérimentales - et pareillement fondée sur la distinction du raisonnement, du témoignage et de l'expérience sensible. C'est dans la deuxième division, que la nouveauté apparaît :

"Les sciences expérimentales peuvent être elles-mêmes divisées en deux séries relativement à l'étude des corps de la nature et à celle des forces qui les meuvent. Les premières peuvent recevoir le nom de sciences matérielles ou naturelles; les secondes, de sciences dynamiques ou physiques $"{ }^{40}$.

Les corps se répartissent, comme dans la première édition, en inorganisés et organisés, les premiers comprenant les astres et les minéraux, les seconds les végétaux et les animaux, l'ensemble de ces dichotomies se trouve présenté sous forme de "tableau synoptique" (cf. fig. l).

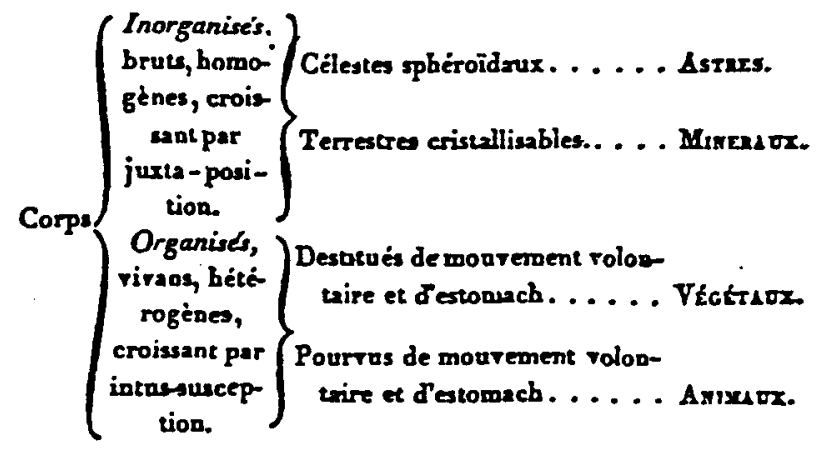

Figure 1. Division des corps

(A.-P. de Candolle, Théorie élémentaire de la botanique, $2^{e}$ éd., Paris, 1819, p. 3).

40. A.-P. de Candolle, op. cit. supra n. 2, 1819, p. 2. 


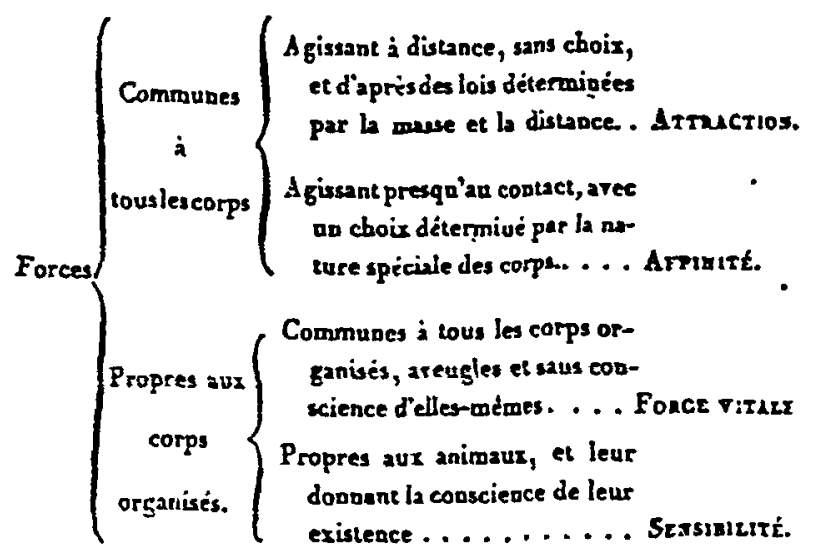

Figure 2. Division des forces (A.P. de CANDOLLE, ibid., p. 4).

Les forces de la nature se disposent selon une série symétrique de la précédente (cf. fig. 2). Elles sont au nombre de quatre. Deux d'entre elles sont communes à tous les corps, l'attraction, "base de tous les phénomènes purement physiques $"$, qui agit sur les corps en fonction de leur masse et de leur distance et indépendamment de leur nature, et l'affinité, «base de tous les phénomènes chimiques " qui intervient entre des corps "placés presque au contact " et diversement selon la nature de chacun. Les deux autres forces sont propres aux corps organisés et elles tendent sans cesse à modifier les précédentes. Il s'agit de la force vitale, «base des phénomènes physiologiques " qui est «commune à tous les êtres organisés ", et de la sensibilité, "base de tous les phénomènes psychologiques (en prenant ce mot dans le sens le plus vaste)" qui est propre aux animaux et qui « leur donne la conscience de leur propre existence ». De ces deux tableaux se déduit $œ$ que de Candolle appelle «l'ordre classique des sciences occupées de l'étude de la nature et considérées dans leurs rapports généraux ". La priorité revient à la physique "qui n'a besoin que du secours des mathématiques $"$, s'applique à tous les corps et constitue la "base commune" des "autres études naturelles». La chimie n'a pu « atteindre quelque précision » que lorsque la physique a été suffisamment développée. De manière analogue la physiologie profite des deux sciences précédentes puisque les corps organisés n'échappent pas à l'attraction et à l'affinité mais voient seulement leur action "modifiée par la force vitale ". Enfin, la psychologie doit recourir à la physiologie pour distinguer ce qui est dû à l'action de la force vitale de ce qui 
revient en propre à la sensibilité. Tout en établissant ainsi entre les sciences dynamiques des "rapports intimes de dépendance ", de Candolle, marque l'autonomie relative de chaque discipline. En effet ce qu'une science peut attendre du progrès des sciences qui la précèdent, c'est essentiellement une délimitation par défaut de son domaine :

« Quand la physique et la chimie auront découvert les lois et les propriétés communes à tous les corps, la physiologie verra clairement ce qui est l'apanage spécial de la force vitale ${ }^{41}$.

La méconnaissance de cette " gradation des sciences dynamiques " a conduit certains "psychologistes" à voir l'action de la sensibilité dans des phénomènes qui relevaient de la force vitale et certains physiologistes à « attribuer à la force vitale ce qui était produit en tout ou en partie, par de simples jeux physiques ou chimiques ». En se référant allusivement à " l'école de Barthez " comme à une illustration de cette illusion, de Candolle se place dans une position de critique nuancée par rapport à l'école de Montpellier et à la tradition vitaliste ${ }^{42}$.

Après ce développement sur les forces de la nature et les sciences dynamiques, les pages consacrées aux corps et aux sciences matérielles ou naturelles, contiennent peu d'éléments nouveaux. Aux astres, aux minéraux, aux plantes et aux animaux, correspondent l'astronomie, la minéralogie, la botanique et la zoologie. Bien que ces disciplines ne présentent pas entre elles de relations de subordination, on peut les placer dans un ordre méthodique, d'après les emprunts qu'elles font aux autres sciences : l'astronomie ne fait appel qu'aux mathématiques et à la physique, la minéralogie y ajoute la chimie. La botanique "qui reçoit des secours de la physique, de la chimie et de la physiologie " et surtout la zoologie, qui fait intervenir en outre la psychologie, présentent une grande complication.

Cette deuxième version de la classification devait satisfaire de Candolle puisqu'il s'y réfère implicitement dans la Physiologie végétale, publiée en 1832, précisant même sa pensée par l'emploi de l'expression «sciences purement descriptives » à propos des sciences matérielles ${ }^{43}$. Nous pou-

41. Ibid., p. 5.

42. Sur l'école de Montpellier, voir Roselyne ReY, Naissance et développement du vitalisme en France, de 1745 à la fin du Premier Empire, thèse de Doctorat d'État, Paris I, 1987.

43. A.-P. de Candolle, Physiologie végétale, Paris, Béchet jeune, 1832, vol. 1, p. 2. 
vons même savoir par le cahier d'un de ses élèves pour l'année 1831-1832 qu'il l'utilisait dans son cours de zoologie ${ }^{44}$.

De Candolle attachait donc quelque importance à cette classification des sciences. Faut-il y voir pour autant la transposition de son activité de botaniste?

\section{L'HISTOIRE NATURELLE ÉLEVÉE AU RANG D'UNE SCIENCE}

On peut, sans risque, penser que la pratique de la systématique végétale avait entraîné de Candolle à manier avec aisance la logique des classifications, mais ce rapprochement trouve rapidement ses limites : la classification des sciences, pour complexe qu'elle soit, est infiniment pauvre par rapport à la classification des espèces végétales qui met en jeu des milliers de taxons. La distinction des méthodes artificielles et des méthodes naturelles, essentielle pour les naturalistes, n'a en matière de classification des sciences qu'une valeur analogique. Si la classification de Jussieu, que défend de Candolle, diffère de celle de Linné, c'est par ses principes mêmes; on pourrait presque dire que « classer » ne s'entend pas dans le même sens pour les uns et les autres. En revanche, la classification des sciences proposée par de Candolle ne diffère pas de celle de Bacon, de celle de d'Alembert ou de celle de Prévost par sa logique mais par la définition de ses objets, et donc en l'occurrence par sa conception de la scientificité.

Cette conception se trouve explicitée dans l'un des premiers textes publiés par de Candolle, l'Essai sur les propriétés médicales des plantes: la science est "l'art de deviner ou de prédire ", définition qu'il faut entendre comme la capacité à « déterminer à l'avance le résultat d'expériences qui n'ont pas encore été faites ${ }^{45}$. Cette définition s'applique fort bien à une discipline qui établit des rapports de cause à effet mais comment peut-elle convenir à une science comme l'histoire naturelle qui semble n'être qu'une « réunion de faits isolés "? La réponse est dans la notion d'organisation et de rapports naturels :

« en étudiant l'organisation, on a reconnu d'abord que certains organes existent ou manquent toujours simultanément, tellement que la présence de

44. In., « Cours de zoologie... ", par Édouard Pictet, Bibliothèque publique et universitaire de Genève, Ms cours universitaire 60 et 61 , référence communiquée par Jean-Daniel Candaux.

45. A.-P. de Candolle, Essai sur les propriétés médicales des plantes, 1804, Paris, 1816, p. 1-2. 
l'un d'eux est un indice assez certains de l'existence des autres; on a reconnu ensuite qu'il est des organes qui exercent sur le reste de la structure une puissance telle, que de la disposition d'une seule partie, on peut déduire la forme de plusieurs autres parties de l'individu; ces deux principes ont fondé la théorie des rapports naturels et de ce moment seul l'histoire naturelle a été élevée au rang d'une science ${ }^{46}$.

L'histoire naturelle, la botanique en particulier, doit tenir son rang de science, telle est la préoccupation qui anime toute l'œuvre d'AugustinPyramus de Candolle. C'est elle qui l'amène à souligner, contre certains naturalistes, que la botanique ne peut plus être une accumulation de faits. C'est elle qui lui fait regretter le dédain de certains physiciens à l'égard d'une discipline qu'ils n'utilisent jamais ${ }^{47}$. C'est elle encore qui explique pourquoi il défend et développe la "méthode naturelle " de classification en botanique, c'est elle enfin qui sous-tend ces classifications des sciences qui lui permettent de situer sa discipline parmi les autres.

Ce n'est pas dans la transposition - banale et limitée - de l'une à l'autre que se rejoignent la classification des espèces et celle des savoirs, mais dans l'unité de leur inspiration, dans la volonté de montrer que l'histoire naturelle à sa manière, différente de la physique, est une science à part entière.

Jean-Marc Drouin, Centre de recherche en histoire des sciences et des techniques, Cité des sciences et de l'industrie, 75930 Paris Cedex 19 (1992).

46. Ibid.

47. A.-P. de Candolle, op. cit. supra n. 6, p. 36. 